\title{
Maxillofacial Radiology 183
}

SADJ August 2020, Vol. 75 No. 7 p395

\section{CJ Nortjé}

Figure 1 is a clinical picture of a thirty year old female who presented with a non-ulcerated swelling which has been present for the last eighteen months. The teeth in the region have been extracted ten months ago but the swelling in the left upper jaw increased in size causing nasal obstruction. Figures 2, 3, 4 and 5 are radiographic images of the swelling. What are the important radiological features and what is your provisional diagnosis?
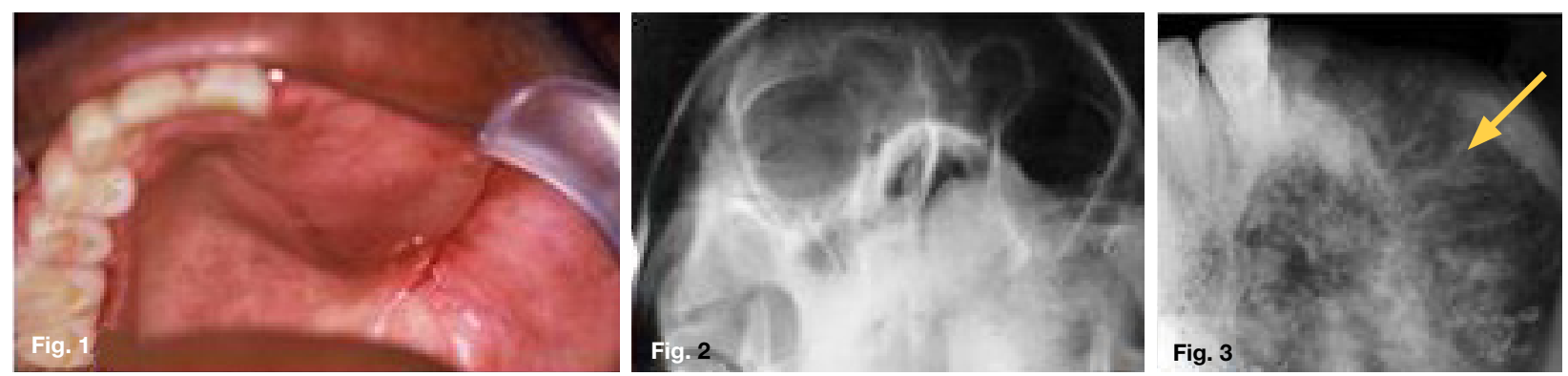


\section{INTERPRETATION}

Looking at the appearance of the remaining teeth in Figure 1 , it is unbelievable that the teeth were extracted due to underlying infection. Patients coming from a remote area where modern medical facilities are not available, teeth are mostly extracted in the presence of a swelling without making a proper diagnosis. Figure 2 shows a mixed radiolucent/radiopacity filling the left maxillary sinus extending into the nasal cavity and causing destruction of the left zygomatic arch. Figure 3 is topographic occlusal view of the maxilla presenting with a "sunray" appearance (yellow arrow). Figure 4 is a CT at the level of the mandibular condyle shows a space occupying calcified lesion in the left maxillary sinus. Figure 5 is a coronal CT showing a mixed radiolucent / radiopaque mass in the left maxilla extending into the adjacent nasal fossa and orbit. A histological diagnosis of a chondrosarcoma was made. The chondrosarcoma is a malignant cartilaginous tumour arising de novo in bone or soft tissue. The tumour is classified as primary or secondary, depending on whether it arises from a

Christoffel J Nortjé: $B C h D, P h D, A B O M R, D S c$. Faculty of Dentistry, University of the Western Cape.

ORCID Number: 0000-0002-9717-5514

Email: cnortje@uwc.ac.za pre-existing benign cartilaginous neoplasm. They are further classified as central (arising inside bone) or peripheral (protruding from bone). Chondrosarcomas com prise $10 \%$ or less of primary malignant bone tumours and occur in patients ranging in age from 16 months to 81 years. In the jaws the peak incidence is in the third to fifth decades. Chondrosarcoma are rare in children. In the maxilla anterior sites is favoured, whereas in the mandible the symphysis, coronoid, condylar processes are affected. The cardinal radiographic signs are a mixed radiolucent/radiopaque lesion with periosteal new bone perpendicular to original cortex so called "sunray appearance". Widening of the periodontal ligament space (red arrow) could be an early sign in chondrosarcoma as well as osteosarcoma (Figure 6). Chondrosarcomas normally present as a non-ulcerated lesion whereas osteo sarcoma present mostly as an ulcerated lesion. In my involvement in maxillofacial radiology over the past forty five years collecting approximately 50 cases, I have never seen any patient recovering from an underlying osteosarcoma or chondrosarcoma. Patients normally die as result of metastasis to the lungs.

\section{Reference}

1. Farman AG, Nortjé CJ, Wood RE: Oral and Maxillofacial Imaging, $1^{\text {st }}$ Ed, Mosby. St. Louis, Missouri 1993; 286-9. 